\title{
Pirfenidone attenuates bladder fibrosis and mitigates deterioration of bladder function in a rat model of partial bladder outlet obstruction
}

\author{
LIU JIAN DUAN, JUN QI, TAO HUANG, XIN GU, DING XU, \\ XIANG JIE KONG and XIAO QIANG QIAN \\ Department of Urology, Xin Hua Hospital Affiliated to Shanghai Jiao Tong University School of Medicine, \\ Shanghai 200092, P.R. China
}

Received July 5, 2014; Accepted March 16, 2015

DOI: $10.3892 / \mathrm{mmr} .2015 .3814$

\begin{abstract}
To investigate the effects of pirfenidone (PFD) on the attenuation of bladder remodeling, and the associated functional changes caused by partial bladder outlet obstruction (pBOO), the present study performed surgery on adult male Sprague-Dawley rats produce a model of pBOO. The rats in the $\mathrm{pBOO}$ group were administered a placebo and, in the CMC group, PFD mixed with the placebo was administered orally at $500 \mathrm{mg} / \mathrm{kg}$ body weight each day for 5 weeks, from 1 week after surgery. The rat bladders were harvested for biochemical analysis following cystometry at the end of the 6 week period. The histopathology was determined using Masson's trichrome staining. The mRNA and protein levels of pro-fibrotic growth factors and extracellular matrix subtypes were assessed. pBOO debilitated bladder function and caused the parameters from cystometry to increase significantly compared with those in the control group $(\mathrm{P}<0.05)$, which were mitigated significantly following PFD treatment $(\mathrm{P}<0.05)$. In terms of the histology, the rats in the $\mathrm{pBOO}$ group exhibited significant increases in bladder weight, muscle hypertrophy and deposition of collagens, which were suppressed by PFD treatment $(\mathrm{P}<0.05)$. Based on the biochemical analysis, significant increases in the mRNA levels of collagen subtypes and growth factors, and protein levels of profibrotic growth factors and $\alpha$-smooth muscle actin in the bladders of rats in the $\mathrm{pBOO}$ group were reduced following PFD treatment. PFD prevented bladder remodeling and attenuated bladder fibrosis and, therefore, mitigated the deterioration of bladder function during the initial stage of $\mathrm{pBOO}$.
\end{abstract}

Correspondence to: Professor Jun Qi, Department of Urology, Xin Hua Hospital Affiliated to Shanghai Jiao Tong University School of Medicine, 1665 Kong Jiang Road, Shanghai 200092, P.R. China E-mail: jasonqi@sh163.net

Key words: bladder fibrosis, extracellular matrix, pirfenidone, partial bladder outlet obstruction, growth factor

\section{Introduction}

Partial bladder outlet obstruction (pBOO) is generally caused by benign prostatic hyperplasia, urethral stricture or urethral congenital malformation. Structurally and functionally, pBOO causes a series of histological and biochemical changes in the bladder wall, through a process of initial inflammation, subsequent hypertrophy and ultimately fibrosis in the decompensation stage (1). This is characterized by increased extracellular matrix (ECM) organization due to collagen synthesis, and increased expression of members of the transforming growth factor (TGF)- $\beta$ family and its receptors in the stroma and epithelium (2). Particularly prevalent during the fibrotic stage of hypertrophy, bladder remodeling couples with alterations in collagen composition (3), with increased expression of type III collagen or a decreased type I/III collagen ratio (4). In addition, various cytokines, chemokines and growth factorsare involved in the development of bladder fibrosis (5).

Pirfenidone (5-methyl-1-phenyl-2-(1H)-pyridone; PFD) is a small molecule pyridone compound. Since its discovery as an antifibrotic agent in a hamster model of bleomycin-induced pulmonary fibrosis (6), the use of PFD in improving organ fibrosis of the lungs $(7,8)$, liver $(9,10)$, kidneys (11), heart (12) and skin (13) have been investigated in vivo or in vitro. Previous studies have demonstrated PFD as a collagen and transforming growth factor (TGF)- $\beta$ production inhibitor, and as an activator of matrix metalloproteinase, thus modulating the fibrogenic pathway (14). It can prevent or reverse the accumulation of extracellular matrix (ECM) by decreasing the expression of profibrotic cytokines, including tumor necrosis factor (TNF)- $\alpha$ and TGF- $\beta$ (15).

Despite improvements in knowledge regarding the pathogenesis of pBOO-induced bladder fibrosis and the different mediators involved, there remains no effective treatment for fibrosis. The present study aimed to examine the effects of PFD on the physiological and histological markers of bladder fibrosis in a rat pBOO model. In addition, the underlying molecular mechanisms were investigated to specifically examine the mRNA and protein expression involved in ECM turnover. It was hypothesized that PFD possessed anti-fibrotic 
properties in bladder wall remodeling, resulting from $\mathrm{pBOO}$ in male rat model, and improve bladder function.

\section{Materials and methods}

Animals and experimental design. All the animal experiments were performed in compliance with the law, approved by the Institutional Animal Care and Use Committee of Xin Hua Hospital (Shanghai, China) (approval no. XHEC-E-2014-016). The rats were housed in a temperature-controlled room in a $12 \mathrm{~h}$ dark/light cycle and had free access to water and food. PFD (cat. no. zc53179-13-8; Chembest Research, Shanghai, China) was mixed with sodium carboxymethyl cellulose (CMC; Beijing Sinopharm Chemical Reagent Co., Ltd., Beijing, China).

A total of 32 adult male Sprague-Dawley rats (Super-B\&K Laboratory Animal Co., Ltd., Shanghai, China), weighing $235 \pm 10 \mathrm{~g}$, were randomly divided into four groups. Group 1 (sham-operation; $\mathrm{n}=8$ ), served as a control, whereas in groups 2-4, surgery was performed to produce pBOO $(n=24)$. In group $30.5 \% \mathrm{CMC}\left(100 \mathrm{mg} / \mathrm{kg}^{-1} / \mathrm{d}^{-1}\right)$ was administered via gavage, as a placebo. In group 4 , PFD mixed with CMC was administered orally at $500 \mathrm{mg} / \mathrm{kg}$ body weight. Drug administration in groups 3 and 4 was performed daily for 5 weeks, beginning 1 week after surgery. The bladders of the rats were harvested for biochemical analysis following cystometry at the end of the 6 week period.

Surgical procedure for $\mathrm{pBOO}$. The $\mathrm{pBOO}$ model was produced in the rats by ligature of the proximal urethra, as previously described (16). In brief, the rats were anesthetized with pentobarbital sodium (Huamaike Biotechnology Co., Ltd., Beijing, China) at a dose of $50 \mathrm{mg} / \mathrm{kg}$ by intraperitoneal injection. An abdominal incision of $\sim 1.5 \mathrm{~cm}$ a was made, and the bilateral prostate was retracted to expose the proximal urethra. A 4-0 silk suture (Ethicon Endo-Surgery, LLC, Cincinatti, OH, USA) was placed around the urethra, and a catheter (BD Biosciences, Franklin Lakes, NJ, USA), measuring $1.10 \mathrm{~mm}$ in outside diameter, was placed adjacent to urethra as a calibration stent. Subsequently, the urethra and catheter were securely ligated and the catheter was carefully removed. Sham operation was performed in the same manner, without ligation of the proximal urethra.

Cystometry. The present study used a previously described cystometry method, with modifications (17). The rats were anesthetized, as mentioned above, and a $2 \mathrm{~cm}$ vertical abdominal incision was made, with the ligature for $\mathrm{pBOO}$ remaining in place. A three-way connection intravenous needle (BD Biosciences) was inserted into the bladder dome with a 6-0 polyethylene suture (Ethicon Endo-Surgery, LLC) fixation. The three-way stopcock was connected to a pressure transducer (Smiths Medical PM, Inc., Waukesha, WI, USA), which was connected to a SurgiVet ${ }^{\circledR}$ Advisor ${ }^{\circledR}$ Vital Signs monitor (Smiths Medical PM, Inc.) to record the intravesical pressure. Any residual urine was collected in order to record the residual urine volume and calculate to zero. The cystometry was performed through a pump (AJ-5803; Angel Electronic Equipments Co., Ltd., Shanghai, China), to infuse room temperature saline (Baxter Healthcare Co., Ltd., Shanghai, China) at a rate of $0.2 \mathrm{ml} / \mathrm{min}$ into the bladder. Following a stabilization period of $30 \mathrm{~min}$, reproducible voiding patterns were achieved and recorded for at least $90 \mathrm{~min}$, due to the different voiding times in the various groups. For each rat, between six and eight voiding cycles were measured to demonstrate consistent bladder behavior. The residual urine was drained and measured following completion of the micturition cycle. The following pressure parameters were measured: Baseline pressure (BP), indicating the lowest bladder pressure during filling; threshold pressure (TP), indicating the pressure just prior to voiding; and the micturition pressure (MP), indicating the maximum pressure during urination. The volume parameters measured included the micturition volume (MV), indicating the residual urine volume and bladder capacity, which was the sum of the residual urine and micturition volumes. Additionally, the micturition interval (MI) was recorded and the bladder compliance was calculated by dividing the mean MV by the difference between the mean TP and mean BP.

Histopathology. Following cystometry, the rat bladder tissues were harvested and weighed. The rats were sacrificed by intraperitoneal injection of $10 \%$ chloral hydrate (Beijing Sinopharm Chemical Reagent Co., Ltd.), following the completion of cystometry and harvesting of the bladder tissue. Half of the harvested tissue was snap frozen in liquid nitrogen (Praxair Co., Ltd., Shanghai, China) for subsequent biochemical analysis. The other half was placed in $4 \%$ paraformaldehyde (Beijing Sinopharm Chemical Reagent Co., Ltd.) for $24 \mathrm{~h}$, and was paraffin-embedded to generate $4 \mu \mathrm{m}$ mid-bladder body transverse sections. The histopathology was examined using Masson's trichrome staining, and 10 randomly selected fields from each bladder section were analyzed using light-microscopy (DMI3000B; Leica Microsystems, Wetzlar, Germany) and a computer-assisted morphometric analyzer (LAS version 3.8.0; Leica Microsystems). The percentage of bladder tissue affected by fibrosis was determined by calculating the ratio of collagen area to smooth muscle area. Quantitative analysis of the collagen area was performed using an image analyzer system (ImageJ 1.34; National Institutes of Health, Bethesda, MA, USA).

Reverse transcription-quantitative polymerase chain reaction $(R T-q P C R)$. Total RNA was isolated from the bladder tissue sections using TRIzol reagent (Takara Bio, Inc., Dalian, China). PrimeScript $^{\mathrm{TM}}$ RT Master mix (cat. no. RR036A; Takara Bio, Inc.) was used to reverse transcribe RNA. Specific primers were designed from their GenBank sequences (http://www. ncbi.nlm.nih.gov/genbank/sequenceids/), synthesized by Sangon Biotech. (Shanghai, China) and are listed in Table I. RT-qPCR was performed using an Applied Biosystems 7500 Fast Real Time PCR system using a SYBR ${ }^{\circledR}$ Premix Ex Taq ${ }^{\mathrm{TM}}$ kit (Takara Bio, Inc.). The PCR cycling conditions were as follows: Initial denaturation, $95^{\circ} \mathrm{C}$ for $30 \mathrm{sec}$; PCR, 40 cycles, $95^{\circ} \mathrm{C}$ for $5 \mathrm{sec}, 60^{\circ} \mathrm{C}$ for $34 \mathrm{sec}$; dissociation stage, $95^{\circ} \mathrm{C}$ for $15 \mathrm{sec}, 60^{\circ} \mathrm{C}$ for $60 \mathrm{sec}$ and $95^{\circ} \mathrm{C}$ for $15 \mathrm{sec}$. The quantity of mRNA was calculated based on the cycle threshold (CT) values, which were standardized against the of the housekeeping gene, GAPDH. The expression levels were determined using the $2^{-\triangle \Delta C T}$ method, with results expressed as the n-fold difference relative to the normal control. 
Table I. Primers used for reverse transcription-quantitative polymerase chain reaction.

\begin{tabular}{|c|c|c|}
\hline Gene & Forward primer $\left(5^{\prime}-3^{\prime}\right)$ & Reverse primer $\left(5^{\prime}-3^{\prime}\right)$ \\
\hline Collagen- $\alpha$ (1) I & ATGTCTGGTTTGGAGAGAGCA (21) & GAGGAGCAGGGACTTCTTGAG (21) \\
\hline Collagen- $\alpha$ (1) III & GCCTCCCAGAACATTACATACC (22) & TTTGCTATTTCCTTCAGCCTTG (22) \\
\hline Elastin & AGTTCCCGGTGGAGTCTATTATC (23) & CAAACGCTCCCAGAAGTCC (19) \\
\hline TGF- $\beta 1$ & CTGCTGACCCCCACTGATAC (22) & CTGTATTCCGTCTCCTTGGTTC (20) \\
\hline CTGF & TGTGAGGAGTGGGTGTGTGAT (21) & AGTTGGCTCGCATCATAGTTG (21) \\
\hline bFGF & GTGTCCATCAAGGGAGTGTGT (21) & CCAACTGGAGTATTTCCGTGA (21) \\
\hline GAPDH & GCAAGTTCAACGGCACAG (18) & GCCAGTAGACTCCACGACAT (20) \\
\hline
\end{tabular}

Numbers in brackets indicate base pairs. TGF, transforming growth factor; CTGF, connective tissue growth factor; bFGF, basic fibroblast growth factor.

Immunohistochemical staining. Slides of the bladder tissues were deparaffinized using xylene (Nanjing Chemical Reagent Co., Ltd., Nanjing, China) and hydrated in graded alcohol solutions (Beijing Sinopharm Chemical Reagent Co., Ltd.). Antigen retrieval by heat mediation was performed using citrate buffer (Beyotime Institute of Biotechnology, Shanghai, China). The slides were then incubated with the primary antibodies: $\alpha$-smooth muscle actin (SMA) rabbit monoclonal antibody (cat. no. 1184-1; Epitomics, Burlingame, CA, USA) diluted 1:1,000 and TGF- $\beta 1$ rabbit polyclonal antibody (cat. no. sc-146; Santa Cruz Biotechnology, Inc., Santa Cruz, CA, USA) diluted 1:100 at $4^{\circ} \mathrm{C}$ overnight. The slides were then incubated with a biotin-conjugated goat anti-rabbit immunoglobulin G (cat. no. MR-SP120; Shanghai Mingrui Biotech Co., Ltd., Shanghai, China), at room temperature. Finally, the slides were incubated with 3,3'-diaminobenzidine substrate and counterstained with hematoxylin. Control sections were incubated without the primary antibody.

Western blot analysis. For western blot analysis, the rat bladder proteins were prepared by homogenizing in radioimmunoprecipitation assay lysis buffer fractions (Beyotime Institute of Biotechnology). Aliquots of the protein extract were transferred onto polyvinylidene fluoride membranes (EMD Millipore, Bedford, MA, USA). Following blocking in 5\% skim milk (Sigma-Aldrich, St Louis, MO, USA), the membranes were incubated with the following primary antibodies overnight at $4^{\circ} \mathrm{C}$ : $\alpha$-SMA rabbit monoclonal antibody $(1: 1,000$; cat. no. 1184-1; Epitomics), TGF- $\beta 1$ rabbit polyclonal antibody (1:100; cat. no. sc-146; Santa Cruz Biotechnology, Inc.), rabbit polyclonal CTGF antibody (1:2,000; cat. no. ab6992; Abcam, Cambridge, MA, USA) and $\beta$-actin (1:5,000; cat. no. A3854; Sigma-Aldrich). The tris-buffered saline containing Tween-20 (Beyotime Institute of Biotechnology) washed membranes were then incubated for $1 \mathrm{~h}$ at room temperature with 1:2,000 secondary antibody conjugated to horseradish peroxidase (cat.no. A0208; Beyotime Institute of Biotechnology). The immunoreactive bands were visualized using enhanced chemiluminescence (EMD Millipore). Densitometric analysis of the bands was performed using Bio-Rad Image $\mathrm{Lab}^{\mathrm{TM}}$ software version 2.0 (Bio-Rad Laboratories, Inc., Hercules, CA, USA). Negative controls were included, omitting the primary antibody. The band intensities were determined using
A

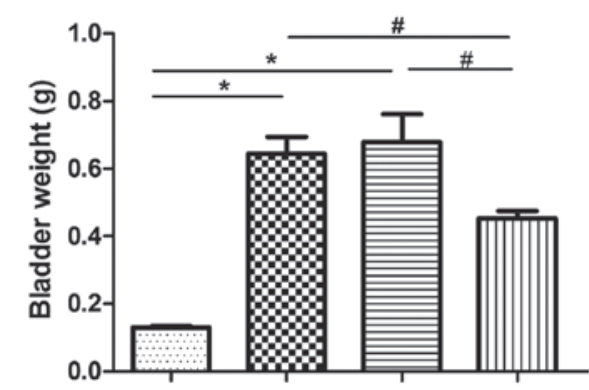

B

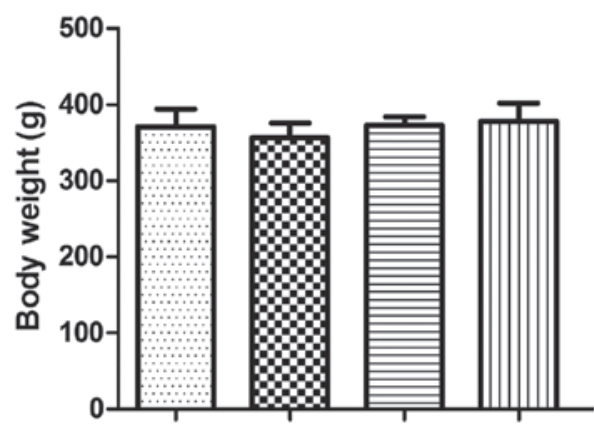

C

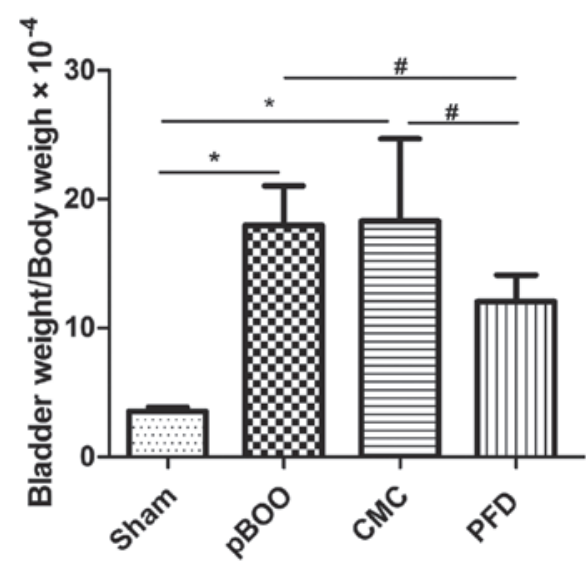

Figure 1. PFD treatment inhibits the increases in bladder weight and bladder weight/body weight ratio. (A) Bladder weight, (B) body weight and $(C)$ bladder weight/body weight ratio of rats in the sham-operation, pBOO, pBOO treated with CMC and $\mathrm{pBOO}$ treated with PFD groups. The obstructed rats exhibited significant increase in bladder weight No significant difference was observed between the $\mathrm{CMC}$ and $\mathrm{pBOO}$ groups, however, PFD treatment significantly inhibited the increase in bladder weight, and in bladder weight/body weight ratio among the groups. Data are presented as the mean \pm standard deviation. " $\mathrm{P}<0.05$, vs. pBOO and CMC groups; ${ }^{\text {"}} \mathrm{P}<0.01$ vs. sham group. $\mathrm{pBOO}$, partial bladder outlet obstruction; CMC, carboxymethyl cellulose; PFD, pirfenidone. 

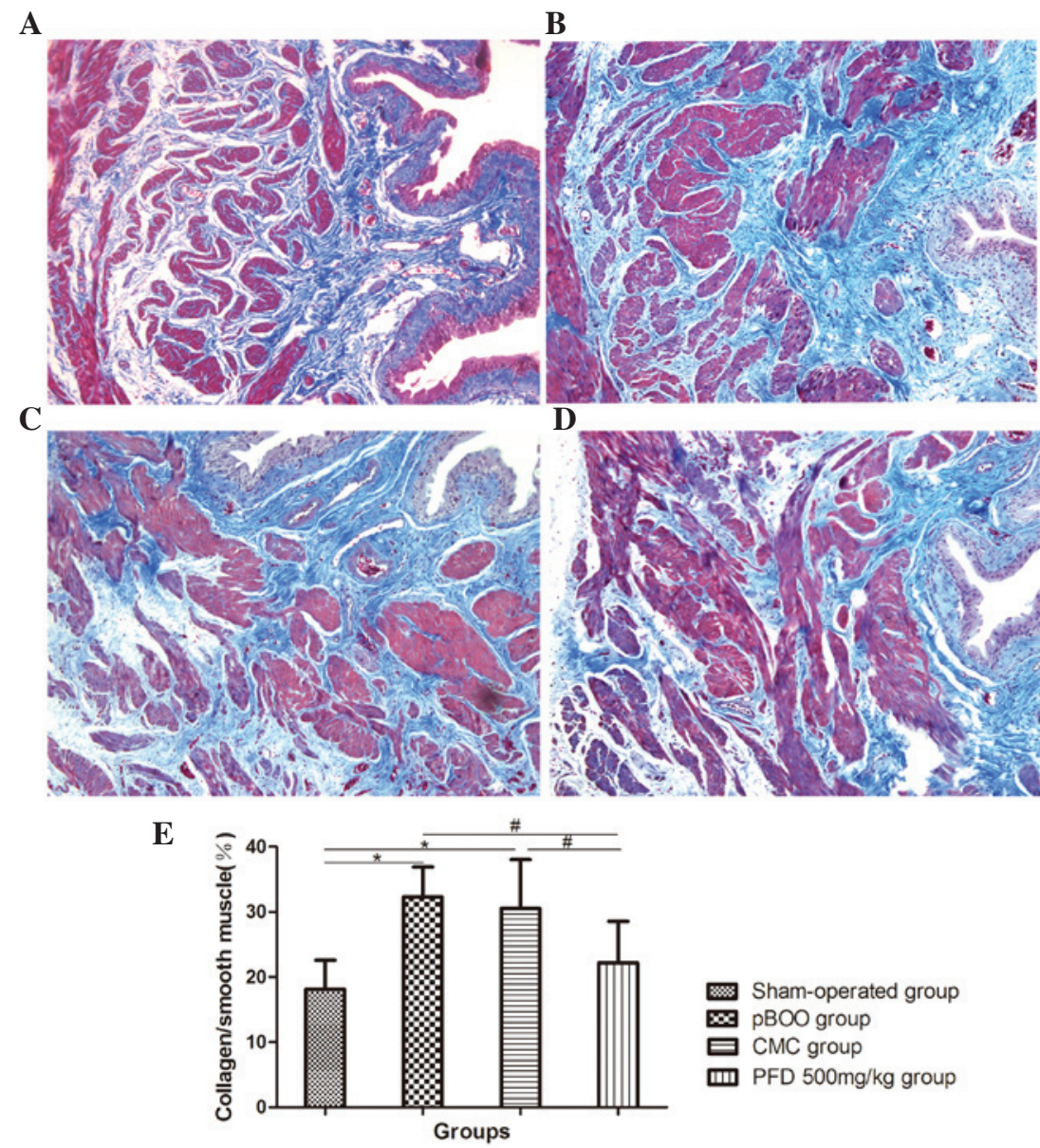

Figure 2. Characterization of rat bladder wall remodeling using Masson's trichrome staining (magnification, x10). Red areas indicate smooth muscle. Blue areas indicate connective tissue. (A) In the sham-operated rat bladder, collagen fiber staining pattern was predominant in the lamina propria, rather than in interfascicular region. (B) In the pBOO rat bladder, detrusor muscle was thickened, and increased collagen content was noted in the lamina propria and interfascicular region. (C) In the CMC bladder, the detrusor muscle was thickened, and increased collagen content was noted in the lamina propria and interfascicular area. (D) In the PFD bladder, smooth muscle hypertrophy was not normalized by PFD treatment, however collagen content was markedly decreased in the lamina propria and interfascicular area. (E) Collagen/smooth muscle ratio was determined using the mean Masson's trichrome staining data in the sham-operated, pBOO, CMC and PFD groups. The obstructed rats exhibited significant increase in collagen/smooth muscle ratio. No significant difference was observed between the placebo group and the $\mathrm{pBOO}$ group, however, PFD treatment significantly inhibited the increase. Data are presented as the mean \pm standard deviation. ${ }^{\sharp} \mathrm{P}<0.05$, vs. $\mathrm{pBOO}$ and $\mathrm{CMC}$ groups; " $\mathrm{P}<0.05$, vs. sham-operated group. pBOO, partial bladder outlet obstruction; CMC, carboxymethyl cellulose; PFD, pirfenidone.

densitometry and the respective intensities were corrected by $\beta$-actin.

Statistical analysis. Data are presented as the mean \pm standard deviation. Groups of data were compared using one-way analysis of variance, followed by least significant difference multiple comparison tests. Analyses were performed using SPSS 17.0 statistical software (SPSS, Inc. Chicago, MO, USA). $\mathrm{P}<0.05$ was considered to indicate a statistically significant difference.

\section{Results}

Predominant findings. Prior to surgery, no significant differences were detected among the groups with regard to body weight. Of the total rats, two were excluded due to bladder rupture. The remaining rats were healthy at the end of the 6 week period. The mean bladder weights were $0.130 \pm 0.012$, $0.640 \pm 0.141,0.679 \pm 0.233$ and $0.453 \pm 0.061 \mathrm{~g}$ for the sham,
pBOO, CMC and PFD groups, respectively. The rats in the pBOO group exhibited significant increases in bladder weight. The bladder weights in the $\mathrm{pBOO}$ group were significantly increased compared with those in the sham group $(\mathrm{P}<0.01)$. No significant difference was found between the $\mathrm{pBOO}$ and CMC groups and, when the ratio of bladder:body weight was normalized, no difference was observed in the ratios between these two groups. The ratios in the $\mathrm{pBOO}$ and CMC groups were significantly higher compared with that the sham group $(P<0.01)$, however, $P F D$ treatment significantly inhibited the increase $(\mathrm{P}<0.05$; Fig. 1$)$.

Cystometry findings. Due to obstruction, the pressure parameters (BP, TP and MP) in the rats in $\mathrm{pBOO}$ and $\mathrm{CMC}$ groups increased compared with the sham group $(\mathrm{P}<0.05)$. In addition, the cystometry in the $\mathrm{pBOO}$ and $\mathrm{CMC}$ groups indicated poor bladder emptying, and increased residual urine volume and micturition interval. A significant increase in the bladder capacity was observed in the $\mathrm{pBOO}$ and $\mathrm{CMC}$ groups 
A

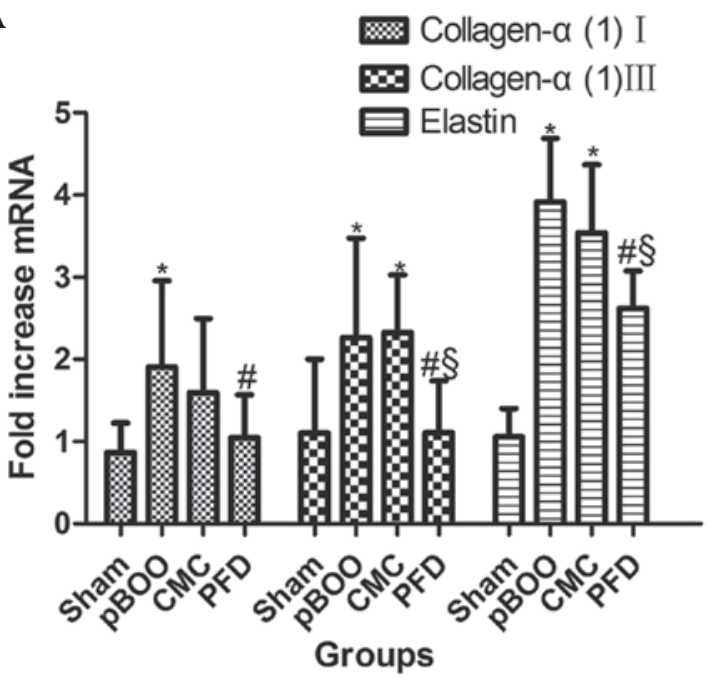

B

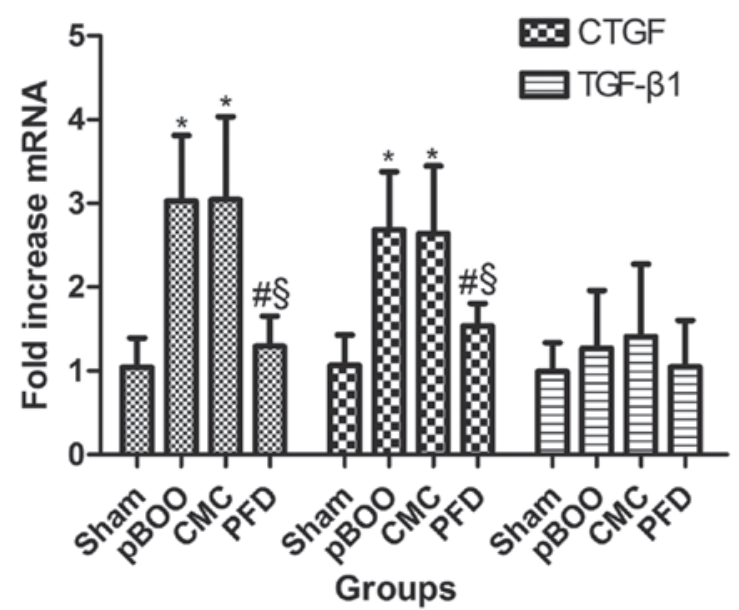

Figure 3. mRNA expression level of collagen subtypes and growth factors. Values are presented as the mean \pm standard deviation of seven or eight individual rats. (A) Gene expression levels of collagen- $\alpha$ (1) I, collagen- $\alpha$ (1) III and elastin of the $\mathrm{pBOO}$ and $\mathrm{CMC}$ groups were higher compared with the sham-operated groups. A significant difference was observed following treatment with PFD compared with the $\mathrm{pBOO}$ and $\mathrm{CMC}$ groups, as it reduced the amplification of collagen- $\alpha$ (1) I, collagen- $\alpha$ (1) III and elastin compared with the pBOO and CMC groups. (B) Gene expression levels of CTGF and bFGF were also higher than in sham-operated group. A significant difference was observed following treatment with PFD, with reduced amplification of CTGF and bFGF compared with the pBOO and CMC groups. Analysis of the gene expression of TGF- $\beta 1$ gene confirmed no significant difference between the obstructed groups and the sham-operated group. ${ }^{*} \mathrm{P}<0.05$, vs. sham-operated group; ${ }^{\prime} \mathrm{P}<0.05$, vs. $\mathrm{pBOO}$ group; ${ }^{8} \mathrm{P}<0.05$, vs. CMC group. TGF, transforming growth factor; $\mathrm{pBOO}$, partial bladder outlet obstruction; CMC, carboxymethyl cellulose; PFD, pirfenidone.

compared with the sham group $(\mathrm{P}<0.05)$. By contrast, TP and MP were decreased in the PFD group compared with the pBOO and $\mathrm{CMC}$ groups $(\mathrm{P}<0.05)$, and a significant decrease in the residual volume and bladder capacity were observed in the PFD group compared with these two groups $(\mathrm{P}<0.05)$, however, PFD treatment did not change the BP, MV, MI or bladder compliance significantly (Table II).

Histopathology. Following Masson's trichrome staining, the collagen was stained blue and muscle was counterstained red. 


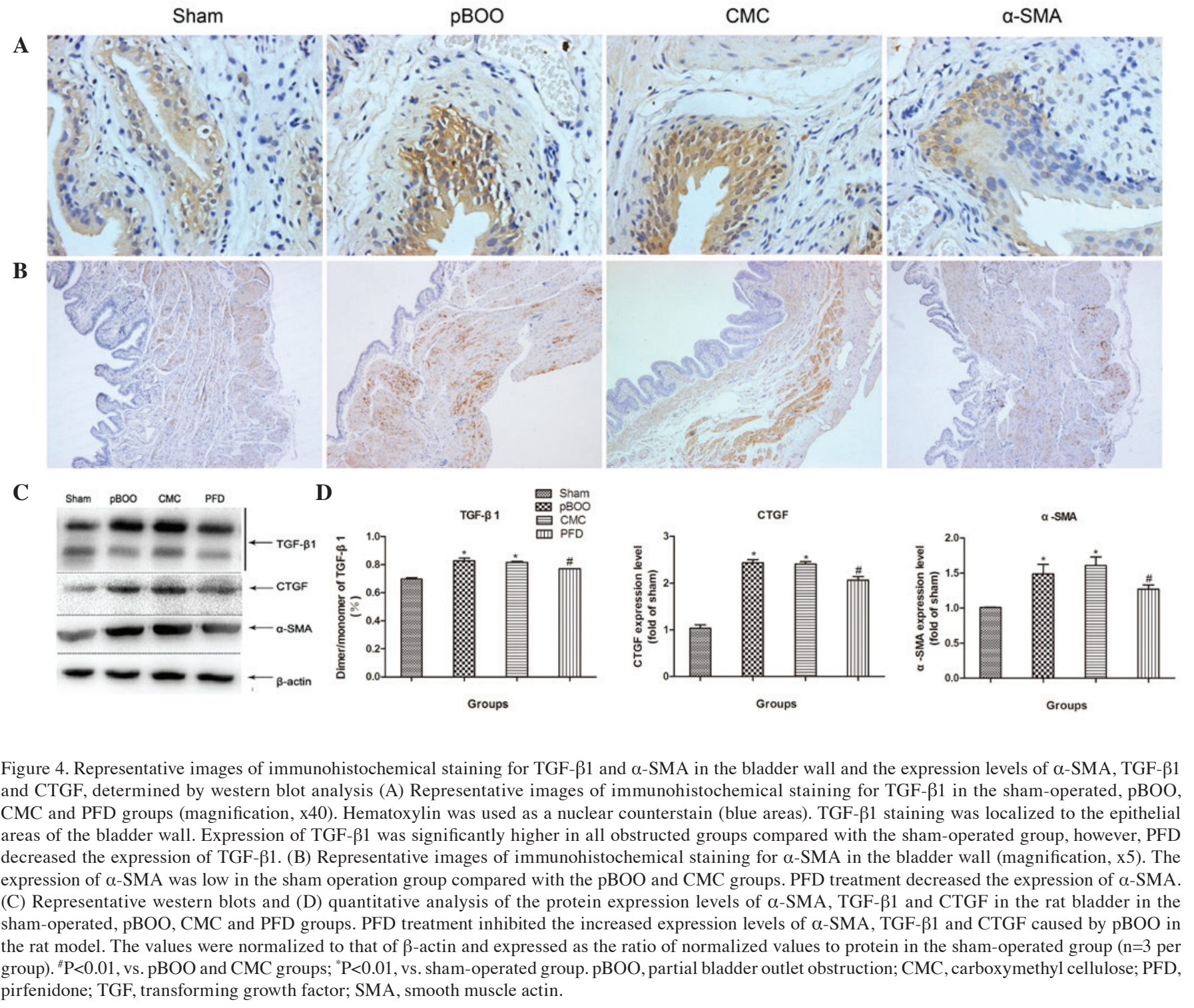

The bladder samples in the $\mathrm{pBOO}$ groups revealed smooth muscle hypertrophy and fibrotic lesions compared with the sham group (Fig. 2A-C). The rats in the $\mathrm{pBOO}$ and $\mathrm{CMC}$ groups exhibited a similar muscle thickness and collagen distribution. pBOO caused hypertrophy of the detrusor muscle and increased the deposition of collagen fibers in the regions of the lamina propria and interfascicular area of the bladder musculature, compared with the sham-operated group. Treatment with PFD treatment following $\mathrm{pBOO}$ bladder did not normalize smooth muscle hypertrophy, however, the collagen content was markedly decreased in the lamina propria and interfascicular area (Fig. 2D). Since collagen deposition was observed in the $\mathrm{pBOO}$ and $\mathrm{CMC}$ groups, the ratio of collagen to smooth muscle was increased, however, PFD treatment inhibited the increase significantly $(\mathrm{P}<0.05$; Fig. $2 \mathrm{E})$.

$R T-P C R$. Collagen- $\alpha$ (1) I, collagen- $\alpha$ (1) III and elastin were significantly upregulated in the $\mathrm{pBOO}$ groups compared with the sham-operated group. Comparison between the groups with regard to PFD treatment revealed a significant difference, with the PFD group exhibiting reduced amplification

of collagen- $\alpha$ (1) I, collagen- $\alpha$ (1) III and elastin compared with the $\mathrm{pBOO}$ and $\mathrm{CMC}$ groups (Fig. 3A). Compared with the sham-operated group, analysis of the gene expression of TGF- $\beta 1$ confirmed no statistically significant increase in the $\mathrm{pBOO}$ and $\mathrm{CMC}$ groups $(\mathrm{P}>0.05)$, however, there was a 2.5 -fold increase in the mRNA expression of CTGF and a 2.9-fold increase in the mRNA expression of bFGF in the pBOO group. Among the operated groups, the amplification of CTGF was significantly lower in the PFD group $(\mathrm{P}<0.01)$. On analyzing the effect of PFD, the gene expression of bFGF in the PFD group was lower compared with the $\mathrm{pBOO}$ and $\mathrm{CMC}$ groups $(\mathrm{P}<0.01)$, whilst no significant difference in expression was observed between the $\mathrm{pBOO}$ and $\mathrm{CMC}$ groups (Fig. 3B).

Immunohistochemical staining and western blot analysis. TGF- $\beta 1$ was detected in the round cells of the bladder endothelium (Fig. 4A1-D1) and $\alpha$-SMA was detected in the bladder lamina propria and smooth muscle cells of the rats in the pBOO group. Hypertrophic smooth muscle cells exhibited increased expression of $\alpha$-SMA compared with those in the sham-operated group, as assessed by immunohistochemistry 
(Fig. 4A2-D2). In terms of the protein expression of TGF- $\beta 1$, all the pBOO groups exhibited a statistically significant increase compared with the sham-operated group, however, PFD treatment promoted a significant downregulation in the pBOO-induced biologically active TGF- $\beta 1$ protein $(\mathrm{P}<0.01)$. Additionally, the protein levels of CTGF and $\alpha$-SMA, assessed using western blot analysis, were higher in the $\mathrm{pBOO}$ and CMC groups compared with the sham group, however, PFD treatment inhibited the protein expression (Fig. 4E and F)

\section{Discussion}

The present study constructed a pBOO model in rats through surgical ligature. The effects on function and bladder remodeling of $\mathrm{pBOO}$ were then analyzed using cystometry and histopathology, respectively. The gene expression levels of pro-fibrotic growth factors and collagen subtypes were examined in rat bladder tissues in which detrusor muscle hypertrophy was induced via pBOO. Additionally, the protein expression levels and distributions were determined using immunohistochemistry and western blot analysis. The effect of PFD in the pBOO model were also examined. The results demonstrated that $\mathrm{PFD}$ reduced the gene expression of pro-fibrotic growth factors and collagen subtypes. These results were in accordance with previous studies reporting anti-fibrotic properties of PFD $(13,18)$.

Due to obstruction, bladder wall remodeling, comprising extensive hypertrophy and increased ECM deposition, occur, all of which cause significant alteration in bladder function. The hypertrophy of bladder smooth muscle cells (BSMCs) and alteration in the architectural organization of collagen in response to $\mathrm{pBOO}$ result in altered $\mathrm{BSMCs} / \mathrm{ECM}$ interactions, which causes a loss of BSMC contractility (19). In the PFD-treated rats, which had received pBOO, the collagen content was markedly decreased in the lamina propria and interfascicular area, however, the hypertrophy of the BSMCs was not normalized by PFD treatment. The composition of ECM is important in maintaining bladder function. Collagen and elastin are the predominant components of ECM, and an increase in their ratios may result in stiffness of the bladder wall and reduced compliance of the bladder (20-22). In the present study, 6 weeks following pBOO, pathological investigations revealed extensive hypertrophy of the BSMCs and deposition of collagen, leading to an increase in bladder weight. In addition, biochemical analysis demonstrated different elevated gene expression levels of collagen subtypes and elastin. As collagen deposition was dominant, the ratio of collagen/ smooth muscle was found o be increased compared with that in the normal bladder. Notably, the present study demonstrated that bladder compliance at the end of the 6 weeks in the pBOO group was increased. A possible explanation for this observation was that the initial increases in the elastin/collagen ratio resulted from the upregulation of elastin production, which was closely paralleled with bladder mechanical compliance. Previous studies have revealed that, in a rat model of spinal cord injury, bladder compliance was increased 3 weeks and 6 weeks after injury, which was closely associated with the collagen/elastin ratio (23). In the present study, bladder compliance was increased 6 weeks after pBOO, on which PFD had no significant effect.
The nature of urinary BSMCs is to remain in a contractile, non-proliferative state, characterized by the expression of h-caldesmon and $\alpha$-SMA. However, urinary BSMCs can become synthetic in response to pathological stimuli. Hypertrophic BSMCs express increased levels of $\alpha$-SMA compared with normal cells (24). In the present study, paraffin sections were immunostained for $\alpha$-SMA, a marker for myofibroblasts and smooth muscle cells. Compared with the sham-operated bladder tissues, the phenotypic changes in the BSMCs of the $\mathrm{pBOO}$ tissues caused a significant increase in the synythesis of $\alpha$-SMA, which suggested a shift to a myofibroblast phenotype, similar to that of fibroblasts in healing wounds and of smooth muscle cells in the cardiovascular system (25). In the PFD-treated pBOO group, $\alpha$-SMA synthesis in the BSMCs was decreased, which was not simply recognized as a direct inhibition of PFD. Since TGF- $\beta$ signaling is reported to be involved in smooth muscle differentiation, it is important to determine the cellular composition of stromal expansion (19).

TGF- $\beta 1$ is a $25 \mathrm{kDa}$ homodimer and heterodimer, present in a wide variety of tissues, which interacts with specific cell membrane receptors and serves as a characterized mediator of cellular phenotype in fibrotic diseases $(26,27)$. In BSMCs TGF- $\beta 1$ induces hypertrophy and the upregulation of collagen (28), and inhibits proliferation (29). In the present study, the hypertrophied rat bladders, produced following urethral constriction exhibited increased urothelial expression of TGF- $\beta 1$, hypertrophy of smooth muscle, and increased expression of type III collagen. This suggested that the TGF- $\beta 1$ from the urothelium acted as a paracrine signal, stimulating the proliferation and matrix production of the BSMCs, thereby contributing to the hypertrophic remodeling of the smooth muscle layer, which was inhibited by PFD treatment. Biologically active TGF- $\beta$ requires dimerization of its monomers and the releases of its latent peptide portion. In the present study PFD was observed to inhibit the increased protein levels of biologically active TGF- $\beta$ caused by pBOO. An important aspect of the antifibrotic mechanism of PFD is associated with its inhibition of the production and activity of TGF- $\beta$ (30).

In addition to antifibrotic properties, the antioxidant (31) and anti-inflammatory activities of PFD have been documented (32), and, although significant attention has been focused on the antifibrotic effect of the drug, the precise mechanism remains to be elucidated. In an animal model of hepatic fibrosis, PFD decreases the mRNA expression levels of TGF- $\beta$ and collagen type 1 (33). Additionally, PFD serves as a novel regulator of the gene expression of cytokines and has the potential to ameliorate established radiation-induced fibrosis (34) A previous study investigating the effects of PFD on secondary progressive multiple sclerosis found that PFD treatment for 20 months not only inhibited the progress of multiple sclerosis, but also significantly improved bladder function (35). PFD has entered the clinical phase of investigation in various fibrotic diseases and the range of doses used fluctuate between 1,800 and 3,600 mg/day. In the present study, a PFD dose of $500 \mathrm{mg} / \mathrm{kg} /$ day, which is a large dose in humans, was used, based on a preliminary experiment that $200 \mathrm{mg} / \mathrm{kg} /$ day of PFD had no significant effects (data not shown) and from previous genotoxic evaluation of 
PFD using an erythrocyte rodent micronucleus assay (36). In addition, due to focusing predominantly on the effect of PFD on the fibrosis-associated mRNA and protein expression of growth factors, the detailed signaling pathway was not further examined. The mechanism of PFD is through its downregulation of a series of cytokines, including TGF- $\beta 1$, CTGF and TNF- $\alpha$ (30). In the present study, no significant difference was observed between the mRNA levles of TGF-- $\beta 1$ in the bladder tissues of the $\mathrm{pBOO}$ rats compared with the sham-operated rats. Increases in the mRNA expression levels of CTGF and bFGF were observed, however, these levels were significantly reduced by PFD, which suggested that PFD inhibited the progression of bladder function via its antifibrotic action.

In the present study, an experimental animal model for obstruction of the bladder neck, induced by ligation, was used. Following construction of the obstruction model, physical examination was performed each day to assure the formation of obstruction and analysis using cystometry was performed while the animals were anesthetized. Urodynamic investigations using pentobarbital sodium may not be optimal, however, the initial objective of the present study was to collect data on bladder pressure, bladder capacity and compliance. In addition, continuous cystometry can be reproducibly performed in anesthetized, non-obstructed rats and in rats with pBOO (37).

In conclusion, the results of the present study support the hypothesis that pirfenidone exhibits anti-fibrosis properties in bladder tissue. These results may provide a future avenue for treatment of bladder fibrosis.

\section{Acknowledgements}

This study was sponsored by the National Natural Science Foundation of China (no. 81070600) and the Foundation of Shanghai Science and Technology Committee (grant nos. 134119a0600 and 14430720800).

\section{References}

1. Metcalfe PD, Wang J, Jiao H, et al: Bladder outlet obstruction: progression from inflammation to fibrosis. Bju Int 106: 1686-1694, 2010.

2. Baskin LS, Sutherland RS, Thomson AA, et al: Growth factors and receptors in bladder development and obstruction. Lab Invest 75: 157-166, 1996.

3. Kim JC, Yoon JY, Seo SI, et al: Effects of partial bladder outlet obstruction and its relief on types I and III collagen and detrusor contractility in the rat. Neurourol Urodyn 19: 29-42, 2000.

4. Kaplan EP, Richier JC, Howard PS, et al: Type III collagen messenger RNA is modulated in non-compliant human bladder tissue. J Urol 157: 2366-2369, 1997.

5. Wick G, Backovic A, Rabensteiner E, et al: The immunology of fibrosis: innate and adaptive responses. Trends Immunol 31: 110-119, 2010.

6. Iyer SN, Margolin SB, Hyde DM and Giri SN: Lung fibrosis is ameliorated by pirfenidone fed in diet after the second dose in a three-dose bleomycin-hamster model. Exp Lung Res 24: $119-132,1998$.

7. Kehrer JP and Margolin SB: Pirfenidone diminishes cyclophosphamide-induced lung fibrosis in mice. Toxicol Lett 90: 125-132, 1997.

8. Azuma A, Nukiwa T, Tsuboi E, et al: Double-blind, placebo-controlled trial of pirfenidone in patients with idiopathic pulmonary fibrosis. Am J Respir Crit Care Med 171: 1040-1047, 2005.
9. Navarro-Partida J, Martinez-Rizo Ab, Gonzalez-Cuevas J, Arrevillaga-Boni G, Ortiz-Navarrete V and Armaendariz-Borunda J: Pirfenidone restricts Th2 differentiation in vitro and limits Th2 response in experimental liver fibrosis. Eur J Pharmacol 678: 71-77, 2012.

10. García L, Hernández I, Sandoval A, et al: Pirfenidone effectively reverses experimental liver fibrosis. J Hepatol 37: 797-805, 2002.

11. Chen JF, Ni HF, Pan MM, et al: Pirfenidone inhibits macrophage infiltration in 5/6 nephrectomized rats. Am J Physiol Renal Physiol 304: F676-F685, 2013.

12. Shi Q, Liu X, Bai Y, et al: In vitro effects of pirfenidone on cardiac fibroblasts: Proliferation, myofibroblast differentiation, migration and cytokine secretion. PLoS One 6: e28134, 2011.

13. Armaendariz-Borunda J, Lyra-Gonzalez I, Medina-Preciado D, et al: A controlled clinical trial with pirfenidone in the treatment of pathological skin scarring caused by burns in pediatric patients. Ann Plast Surg 68: 22-28, 2012.

14. Zhao XY, Zeng X, Li XM, et al: Pirfenidone inhibits carbon tetrachloride- and albumin complex-induced liver fibrosis in rodents by preventing activation of hepatic stellate cells. Clin Exp Pharmacol Physiol 36: 963-968, 2009.

15. Iyer SN, Gurujeyalakshmi G and Giri SN: Effects of pirfenidone on transforming growth factor-beta gene expression at the transcriptional level in bleomycin hamster model of lung fibrosis. J Pharmacol Exp Ther 291: 367-373, 1999.

16. Liu F, Yao L, Yuan J, et al: Protective effects of inosine on urinary bladder function in rats with partial bladder outlet obstruction. Urology 73: 1417-1422, 2009.

17. Kim JW, Jang HA, Bae JH and Lee JG: Effects of coenzyme Q10 on bladder dysfunction induced by chronic bladder ischemia in a rat model. J Urol 189: 2371-2376, 2013.

18. Oku H, Shimizu T, Kawabata T, et al: Antifibrotic action of pirfenidone and prednisolone: different effects on pulmonary cytokines and growth factors in bleomycin-induced murine pulmonary fibrosis. Eur J Pharmacol 590: 400-408, 2008.

19. Parekh A, Long RA, Chancellor MB and Sacks MS: Assessing the effects of transforming growth factor-betal on bladder smooth muscle cell phenotype. II. Modulation of collagen organization. J Urol 182: 1216-1221, 2009.

20. Cortivo R, Pagano F, Passerini G, et al: Elastin and collagen in the normal and obstructed urinary bladder. Br J Urol 53: 134-137, 1981.

21. Ewalt DH, Howard PS, Blyth B, et al: Is lamina propria matrix responsible for normal bladder compliance? J Urol 148: 544-549, 1992.

22. Djavan B, Lin V, Kaplan EP, et al: Decreased elastin gene expression in noncompliant human bladder tissue: a competitive reverse transcriptase-polymerase chain reaction analysis. J Urol 160: 1658-1662, 1998.

23. Toosi KK, Nagatomi J, Chancellor MB and Sacks MS: The effects of long-term spinal cord injury on mechanical properties of the rat urinary bladder. Ann Biomed Eng 36: 1470-1480, 2008.

24. Bai J, Liu XS, Xu YJ, Zhang ZX, Xie M and Ni W: Extracellular signal-regulated kinase activation in airway smooth muscle cell proliferation in chronic asthmatic rats. Sheng Li Xue Bao 59: 311-318, 2007.

25. Schultz K, Murthy V, Tatro JB and Beasley D: Endogenous interleukin-1 alpha promotes a proliferative and proinflammatory phenotype in human vascular smooth muscle cells. Am J Physiol Heart Circ Physiol 292: H2927-H2934, 2007.

26. Runyan CE, Poncelet AC and Schnaper HW: TGF-beta receptor-binding proteins: complex interactions. Cell Signal 18: 2077-2088, 2006.

27. Leask A and Abraham DJ: TGF-beta signaling and the fibrotic response. FASEB J 18: 816-827, 2004.

28. Howard PS, Kucich U, Coplen DE and He Y: Transforming growth factor-beta1-induced hypertrophy and matrix expression in human bladder smooth muscle cells. Urology 66: 1349-1353, 2005.

29. Barendrecht MM, Mulders AC, van der Poel H, et al: Role of transforming growth factor beta in rat bladder smooth muscle cell proliferation. J Pharmacol Exp Ther 322: 117-122, 2007.

30. Schaefer CJ, Ruhrmund DW, Pan L, et al: Antifibrotic activities of pirfenidone in animal models. Eur Respir Rev 20: 85-97, 2011.

31. Salazar-Montes A, Ruiz-Corro L, López-Reyes A, et al: Potent antioxidant role of pirfenidone in experimental cirrhosis. Eur J Pharmacol 595: 69-77, 2008.

32. Oku H, Nakazato H, Horikawa T, et al: Pirfenidone suppresses tumor necrosis factor-alpha, enhances interleukin-10 and protects mice from endotoxic shock. Eur J Pharmacol 446: 167-176, 2002. 
33. Tada S, Nakamuta M, Enjoji M, et al: Pirfenidone inhibits dimethylnitrosamine-induced hepatic fibrosis in rats. Clin Exp Pharmacol Physiol 28: 522-527, 2001.

34. Simone NL, Soule BP, Gerber L, et al: Oral pirfenidone in patients with chronic fibrosis resulting from radiotherapy: a pilot study. Radiat Oncol 2: 19, 2007.

35. Walker JE, Giri SN and Margolin SB: A double-blind,randomized, controlled study of oral pirfenidone for treatment of secondary progressive multiple sclerosis. Mult Scler 11: 149-158, 2005.
36. Alcántar-Díaz BE, Gómez-Meda BC,Zúñiga-González GM, et al: Genotoxic evaluation of pirfenidone using erythrocyte rodent micronucleus assay. Food Chem Toxicol 50: 2760-2765, 2012.

37. Pandita RK, Fujiwara M, Alm P and Andersson KE: Cystometric evaluation of bladder function in non-anesthetized mice with and without bladder outlet obstruction. J Urol 164: 1385-1389, 2000. 